\title{
Effective learning strategies and schedule for acquiring new patterns of bimanual coordination
}

\author{
Seijiro Tsutsui* \\ Department of Physical Education, Aichi University of Education, Japan
}

Human movements are often attracted into particular movement patterns by some neural, musculo-skeletal, and biomechanical constraints in spite of performer's intention. Such attracted patterns are called "attractors". Acquisition of a skilled movement often needs to overcome the attractors. Although most learners who want to acquire such tasks tend to be subject to the attractors in the early stage of practice, they gradually become to overcome the attractors as the practice proceeds, and finally enable to perform the desired movement. However, detailed learning processes and effective learning methods for acquiring a new movement have not yet been elucidated. Therefore, the present study examined both what type of learning strategies and what type of learning schedule are effective for overcoming the attractors and thus acquiring a new movement effectively.

For the above purpose, a bimanual coordination task which involved continuous back-and-forth movements of both upper limbs whereby one limb led the other limb by $1 / 4$ of a complete cycle $(90$ degree relative phase) was employed as an experimental task in the present study. In the early stage of practice, even when learners try to perform the 90 degree relative phase, they are normally attracted either to the in-phase, in which adduction and abduction of both upper limbs are synchronized in a symmetric form, or anti-phase, in which adduction of one upper limb is synchronized, in a reciprocal form, with adduction of the other upper limb. Subjects in the present study were required to overcome either the in-phase or anti-phase so as to acquire the 90 degree relative phase.

Three learning strategies, forced response method, observational learning, and advanced organization, were examined in Chapter 3 of the present study by conducting four experiments. The three learning strategies were compared in Experiment 1. Both the forced response method and the observational learning were then respectively examined in detail in Experiments 2 and 3. In Experiment 4, both skill level and presentation schedule in the observational learning were further examined.

In Experiment 1, a forced response method group was guided by an expert in some trials; an observational learning group observed performance made by an expert; an advanced organization group was given information about the meaning and structure of augmented feedback before learning; and a control group performed usual physical practice alone. Although all the four groups were given augmented feedback after each trial, the advanced organization group alone was given information about the meaning and structure of augmented feedback. In a retention test conducted in a week after acquisition trials, the advanced organization group showed significantly better performance than the other three groups did. Therefore, it was shown that the advanced organization was an effective strategy. Presentation of such a conceptual clue given in advance for the advanced organization group seems to bring about a cognitive foothold for effective learning and can thus promote acquisition of a new movement. Furthermore, the forced response method group showed significantly worse performance than the control group did. This is inconsistent with the Schmidt-Lee (1999) assumption that the forced response method can be effective in a task which learners do not know how to do. The observational learning group showed larger errors than the control group, although the difference did not reach significant level.

The finding of Experiment 1 that both the forced response method group and the observational learning group did not show better performance than the control group may be explained in terms of the less amount of physical practice trials. Both groups performed either guidance trials or observational trials instead of a part of physical practice trials. Thus both the forced response method and the observational learning were then respectively examined in detail in Experiments 2 and 3. In Experiment 2, four groups, mixed group (this was exactly the same as the guidance group in Experiment 1 , and the total number of trials was the same as a control, physical practice group), augmented guidance group (the physical practice plus guidance), guidance only group (no physical practice), and physical practice group (this was exactly the same as the control group in Experiment 1), were compared. The main result indicated that the guidance only group showed significantly worse performance than the other three groups, with no difference between the three groups.

In Experiment 3, four groups, mixed group (this was exactly the same as the observational learning group in Experiment 1, and the total number of trials was the same as a control, physical practice group), augmented observation group (physical practice plus observational learning), observation only group (no physical practice), and physical practice group (this was exactly the same as the control group in Experiment 1), were compared as in Experiment 2. The result also indicated that the observation only group showed significantly worse performance than the other three groups, with no difference between the three groups. Both experiments thus showed that both the forced response method and the observational learning were not effective when learners could perform physical practice.

Experiment 3 showed that the observational learning was not effective in motor learning. This may have resulted from the difference

Correspondence to: Seijiro Tsutsui, Department of Physical Education, Aichi University of Education, Japan, Tel: +81-566-26-2462/ +81-561-36-5555, E-mail: stsutsui@auecc.aichi-edu.ac.jp / tsutsui-s@tokaigakuen-u.ac.jp

Received: February 11, 2018; Accepted: February 26, 2018; Published: February 28,2018 
in skill level between the model used in the observational learning and the learners. The model's skill level was therefore examined with two different types of presentation schedule in Experiment 4. Two models, an expert model who showed skillful performance throughout observational learning trials and a learning (novice) model who showed whole acquisition processes of learning, were employed. Two types of presentation schedules, an alternate schedule in which learners alternately performed physical practice trials and observational trials and a concentrated schedule in which learners consecutively performed all observational trials before physical practice sessions, were employed. Thus, five groups, control group (without any observational learning), concentrated-expert group (concentrated observation of the expert model), alternate-expert group (alternate observation of the expert model), concentrated-learning group (concentrated observation of the learning model), and alternate-learning group (alternate observation of the learning model), were compared. The result indicated that the concentrated-learning group showed significantly better performance than any of the concentrated-expert group, the alternate-expert group and the control group, suggesting that the concentrated presentation of a learning model was most effective in observational learning.

Furthermore, learning schedule was also examined in Experiments 5 and 6 in the present study. Two learning schedules, the blocked practice, in which learners consecutively performed a single movement pattern alone, then performed another pattern, and so on, and the random practice, in which learners randomly performed several movement patterns even when they could not well performed each pattern. The random practice showed significantly better performance than the blocked practice in a retention test, whereas the blocked practice showed better performance than the random practice in acquisition trials. This phenomenon is called the contextual interference. The previous study of Newell and McDonald (1992) pointed out that the contextual interference effect may be limited only for the tasks that involve rescaling processes of a previously learned movement pattern, and that it may not be applicable to learning tasks that require the learners to acquire a new coordination pattern as used in the present experiment. However, the findings in Experiments 5 and 6 denied this notion and showed evidence for the contextual interference effect in learning a new coordination pattern.

These findings on the strategies and schedules in learning a new coordination pattern differed in some points from those in the learning of movement parameters, such as force and timing, which are used in modifying a previously acquired movement pattern. Thus, the previous theories of motor learning, such as the schema theory (which is developed on the basis of findings on parameter learning), may not explain the present findings.

Main points of the discussion in the present study were as follows. First, a most suitable strategy for the bimanual coordination task was shown to be a combination of advanced organization and actual physical practice. This was explained to mean that the advanced organization provided in advance the learners with whole insight into the learning processes. Second, the concentrated presentation of a learning model was found to be most effective in observational learning. It was likely that the learning model demonstrated progresses from an initial learning stage in which the learning model could not perform anything at all to the final stage in which the learning model became an expert in performing the task. The use of a learning model may thus have provided information about problem-solving processes as well as enhanced learners' motivation, because both the learning model and learners gradually improved their performance as the practice proceeded. These findings on learning strategies therefore suggested that the coordination learning needs an engagement of learners to cognitive factors subserving motor learning more than the parameter learning, even though the task used in coordination learning is largely influenced by the intrinsic attractors. Third, Chapter 4 demonstrated that the contextual interference effects occurred in the coordination learning as well as parameter learning. The random practice schedule was clearly shown to promote acquiring the new bimanual coordination pattern better than the blocked practice schedule. The random practice may have forced the learners to reconstruct memories of motor patterns or programs during the random practice and accordingly elaborated memory processes more than during the blocked practice. This also seemed to be consistent with the findings of learning strategies that cognitive factors facilitate the coordination learning, despite the selforganizing nature of the bimanual coordination task. Finally, although the experiments suggested that learners can use specific effective learning strategies and learning schedules in the motor learning of a new bimanual coordination pattern, the effective strategies and schedules per se could not be useful without physical practice.

Copyright: (C2018 Tsutsui S. This is an open-access article distributed under the terms of the Creative Commons Attribution License, which permits unrestricted use, distribution, and reproduction in any medium, provided the original author and source are credited. 